\title{
Efecto de la materia orgánica y la capacidad de intercambio catiónico en la acidez de suelos cultivados con maíz en dos regiones de Chiapas, México Effect of organic matter and cation exchange capacity on the acidity of soils cultured with corn in two regions of Chiapas, Mexico
}

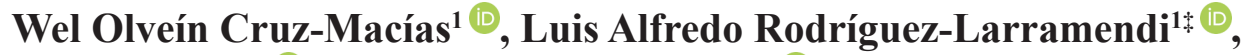 \\ Miguel Ángel Salas-Marina ${ }^{1}{ }^{\circledR}$, Vidal Hernández-García ${ }^{1}{ }^{\circledR}, \operatorname{Rady}_{\text {Alejandra Campos-Saldaña }}{ }^{\circledR}$, \\ Moisés Hussein Chávez-Hernández ${ }^{1}{ }^{\circledR}$ y Alder Gordillo-Curiel ${ }^{1}{ }^{\circledR}$
}

\footnotetext{
${ }^{1}$ Universidad de Ciencias y Artes de Chiapas, Fac. de Ingeniería, Sede Villa Corzo. Carretera VillaCorzo a ejido Monterrey km 3. 30520 Villacorzo, Chiapas, México.

*Autor para correspondencia (alfredo.rodriguez@unicach.mx)
}

\section{RESUMEN}

La tercera parte de los suelos del mundo son ácidos, en estas condiciones muchos cultivos se afectan, sobre todo cuando las concentraciones de aluminio alcanzan niveles tóxicos. Con el objetivo de establecer las relaciones entre indicadores de acidez y la capacidad de intercambio catiónico, el $\mathrm{pH}$ y el contenido de materia orgánica, se seleccionaron 13 sitios en las regiones Frailesca y Valles Zoque. Se tomaron cinco submuestras a $0.30 \mathrm{~m}$ de profundidad, para luego conformar una muestra homogénea por sitio experimental. Se determinaron el $\mathrm{pH}$, cationes intercambiables, capacidad de intercambio catiónico, contenido de materia orgánica, saturación de aluminio $\left(\mathrm{Al}^{3+}\right)$ y de hidrógeno $\left(\mathrm{H}^{+}\right)$. El porcentaje de saturación del $\mathrm{Al}^{3+}$ se correlacionó exponencialmente con incrementos en la CIC, el pH y el \% de saturación del calcio $\left(\mathrm{Ca}^{2+}\right)$; $\sin$ embargo, respecto a la materia orgánica, la saturación de $\mathrm{Al}^{3+}$ se ajustó significativamente a un modelo polinomial de segundo grado, indicando un máximo de saturación a valores de $4.14 \%$ de materia orgánica. El porcentaje de saturación de $\mathrm{H}^{+}$se ajustó negativamente a una función exponencial, disminuyendo a valores de materia orgánica superiores a $2 \%$. Se concluye que en los suelos de la región Frailesca se está produciendo un proceso de acidificación que pudiera conducir a incrementos tóxicos del aluminio para el cultivo de maíz, sobre todo a valores de $\mathrm{pH}$ inferiores a cinco.

Cita recomendada:

Cruz-Macías, W. O., L. A. Rodríguez-Larramendi, M Á. Salas-Marina, V. Hernández-García, R. A. Campos-Saldaña, M. H. Chávez-Hernández ${ }^{1}$ y A. Gordillo-Curiel. 2020. Efecto de la materia orgánica y la capacidad de intercambio catiónico en la acidez de suelos cultivados con maíz en dos regiones de Chiapas, México. Terra Latinoamericana 38: 475-480.

DOI: https://doi.org/10.28940/terra.v38i3.506
Tales incrementos en la acidificación de los suelos pudieran estar asociados al excesivo uso de fertilizantes amoniacales producto de una agricultura mucho más intensiva que en la región Valles Zoques.

Palabras clave: acidez intercambiable, aluminio, fertilidad del suelo, suelos ácidos.

\section{SUMMARY}

One third of the world's soils are acidic, under these conditions many crops are affected, especially when aluminum concentrations reach toxic levels. In order to establish the relationships between acidity indicators and cation exchange capacity, and $\mathrm{pH}$ and organic matter content, 13 sites were selected in Frailesca and Valles Zoque regions. Five sub-samples were collected at a depth of $0.30 \mathrm{~m}$, in order to form a homogeneous sample per experimental site. Determination of $\mathrm{pH}$, interchangeable cations, cation exchange capacity, organic matter content, and aluminium $\left(\mathrm{Al}^{3+}\right)$ and hydrogen $\left(\mathrm{H}^{+}\right)$saturation was performed. The saturation percentage of $\mathrm{Al}^{3+}$ was exponentially correlated with increases in $\mathrm{CIC}, \mathrm{pH}$ and calcium $\left(\mathrm{Ca}^{2+}\right)$ saturation \%; however, in regard to organic matter, $\mathrm{Al}^{3+}$ saturation adjusted significantly to a second order polynomial model, indicating a maximum saturation at values of $4.14 \%$ of organic matter. The percentage of $\mathrm{H}^{+}$ saturation adjusted negatively to an exponential 
function, decreasing at organic matter values higher than $2 \%$. It is concluded that in the soils of Frailesca region, there is an acidification process that could lead to toxic increases of aluminum for corn culture, especially at $\mathrm{pH}$ values lower than five.

Index words: interchangeable acidity, aluminum, soil fertility, acid soils.

\section{INTRODUCCIÓN}

En el mundo, el $30-40 \%$ de los suelos cultivables y hasta el $70 \%$ de los considerados potencialmente cultivables son ácidos. En estos suelos, el ion aluminio activo $\left(\mathrm{Al}^{3+}\right)$ se considera el factor más importante que limita el crecimiento de las plantas, aunque existen otros factores restrictivos, como la deficiencia de fósforo $(\mathrm{P})$, calcio $(\mathrm{Ca})$, magnesio $(\mathrm{Mg})$ y manganeso tóxico (Muhammad et al., 2019).

Ante las limitaciones que ejerce la acidez del suelo y en particular la toxicidad por aluminio en el crecimiento de las plantas, se ha estudiado el papel que ejerce la materia orgánica. En este sentido Zhao et al. (2020), demostraron el efecto que ejercen diferentes enmiendas orgánicas aplicadas al suelo con diferentes niveles de toxicidad por aluminio. Los resultados mostraron que todas las enmiendas disminuyeron significativamente las concentraciones totales de $\mathrm{Al}$, $\mathrm{Al}$ monomérico, $\mathrm{Al}$ monomérico inorgánico y $\mathrm{Al}^{3+}$ en la solución del suelo. Las aplicaciones de desechos alcalinos y cal fueron más efectivas para disminuir las concentraciones de especies de Al fitotóxicas en la solución del suelo que las aplicaciones de los tres materiales orgánicos solos (Zhao et al., 2020).

La acidificación del suelo inhibe el crecimiento microbiano y conduce a la disminución de la abundancia y diversidad de los microbios del suelo (Wan et al., 2019), lo que reduce aún más la estabilidad espacial de los ecosistemas de los suelos agrícolas, sobre todo en aquellos con bajos contenidos de materia orgánica. Esto trae consigo cambios en las comunidades de depredadores de insectos y la disminución de los controles biológicos, lo que reduce la sostenibilidad de la producción agrícola (Lal, 2015). Otros estudios han indicado que la disminución del rendimiento de los cultivos en suelos ácidos está relacionada con la disminución de la función bacteriana del suelo (Lupwayi et al., 2014; Wan et al., 2019).
Por otra parte, es importante conocer cómo se modifica la capacidad de intercambio catiónico (CIC) en suelos ácidos. La CIC es la carga eléctrica negativa de las arcillas y materia orgánica del suelo y puede ser permanente o dependiente del $\mathrm{pH}$, en función del tipo de arcilla (López-Báez et al., 2019). Su importancia radica en que con ella se puede conocer el porcentaje de saturación de bases o cantidad relativa de bases en el suelo lo cual determina su fertilidad y se ha demostrado que en los suelos con menos de $25 \%$ de arcilla y mayor contenido de MO, la CIC también es influenciada por el $\mathrm{pH}$ del suelo.

Ante este fenómeno de acidificación paulatina de los suelos dedicados al cultivo de maíz, particularmente en la región Frailesca de Chiapas, los cuales presentan una baja fertilidad evidenciada por su generalizada acidez y bajo contenido de materia orgánica (LópezBáez et al., 2019), se realizó el presente estudio con el objetivo de establecer las relaciones entre indicadores de acidez y la capacidad de intercambio catiónico, el $\mathrm{pH}$ y el contenido de materia orgánica de suelos cultivados con maíz en las regiones Frailesca y Zoques de Chiapas.

\section{MATERIALES Y MÉTODOS}

\section{Localización}

La investigación se realizó durante meses de julio a noviembre de 2016 en 13 sitios ubicados en comunidades de las regiones Frailesca y Valles Zoques de Chiapas (Figura 1). La selección de las regiones y los sitios de estudio se realizó teniendo en cuenta la importancia del cultivo de maíz y la contribución que realiza la región Frailesca a la producción del cereal del estado y las características actuales de degradación, particularmente la acidificación y compactación de los suelos, así como el contraste en tipo de suelo y manejo agronómico del cultivo de maíz en ambas regiones. E1 clima predominante en la región Valles Zoque es cálido subhúmedo con lluvias de verano. Durante los meses de mayo a octubre, la precipitación pluvial oscila de los 800 a $2300 \mathrm{~mm}$. Los suelos predominantes son los litosoles y luvisoles. Los suelos litosoles ocupan la mayor parte de la sierra alta de laderas tendidas, en el centro y este de la región. Son suelos con menos de $10 \mathrm{~cm}$ de espesor, que generalmente sostienen una vegetación baja, a los que se les conoce también como leptosoles, 
con textura y colores variables, predominando en ella la materia orgánica (INEGI, 2017). La región Frailesca presenta climas de los grupos cálidos y semicálidos. Predomina el cálido subhúmedo con lluvias en verano. Durante los meses de mayo a octubre, la precipitación pluvial oscila de los $1000 \mathrm{~mm}$ y hasta los $2600 \mathrm{~mm}$. Presenta diez unidades de suelo, siendo las principales: litosol, regosol y acrisol (INEGI, 2017).

\section{Diseño de Muestreo y Análisis Químicos}

Durante el mes de junio de 2016, en parcelas que variaron entre 0.5 a 1 ha, de acuerdo con la disponibilidad de terreno cultivado con maíz de cada productor, se tomaron cinco muestras de suelo de 0 a $0.30 \mathrm{~m}$, por ser la profundidad donde se encuentra el mayor volumen de raíces (Montejo-Martínez et al., 2018). Con las cinco muestras se conformó una muestra homogénea por sitio y se enviaron al laboratorio Fertilizab $^{\circledR}$ donde se realizaron los análisis de acuerdo con la Norma Oficial Mexicana (SEMARNAT, 2002) para medir $\mathrm{pH}$, capacidad de intercambio catiónico total (CIC), porcentaje de saturación de cationes intercambiables, contenido de materia orgánica (MO) y acidez intercambiable $\left(\mathrm{Al}^{3+} \mathrm{y} \mathrm{H}^{+}\right)$.

\section{Análisis Estadístico}

Se realizaron análisis de regresión, lineales y no lineales $(P \leq 0.05)$, para evaluar el efecto del contenido de materia orgánica y el $\mathrm{pH}$ sobre la capacidad de intercambio catiónico (CIC), así como el efecto de la CIC, la materia orgánica, el pH y el \% de saturación por $\mathrm{Ca}^{2+}$ en la saturación por $\mathrm{Al}^{3+}$. También se evaluó la dependencia entre el $\mathrm{H}^{+}$intercambiable y el contenido de materia orgánica. Los análisis se realizaron con el software STATISTICA ${ }^{\circledR}$ (StatSoft, 2015).

\section{RESULTADOS Y DISCUSIÓN}

Se observó una relación exponencial negativa entre el porcentaje de saturación de aluminio $\left(\mathrm{Al}^{3+}\right)$ y la capacidad de intercambio catiónico, el pH y el calcio $\left(\mathrm{Ca}^{2+}\right)$ intercambiable (Figura 2), en los tres casos con coeficientes de determinación $\left(\mathrm{r}^{2}\right)$ iguales o superiores al $40 \%$, siendo más estrecha dicha dependencia con el porcentaje de $\mathrm{Ca}^{2+}$, con un coeficiente de determinación de $82 \%$. Se debe destacar que los mayores valores de saturación de aluminio se presentaron en los suelos de la región Frailesca (datos no mostrados), lo cual constituye una alerta ante la acidificación de los suelos

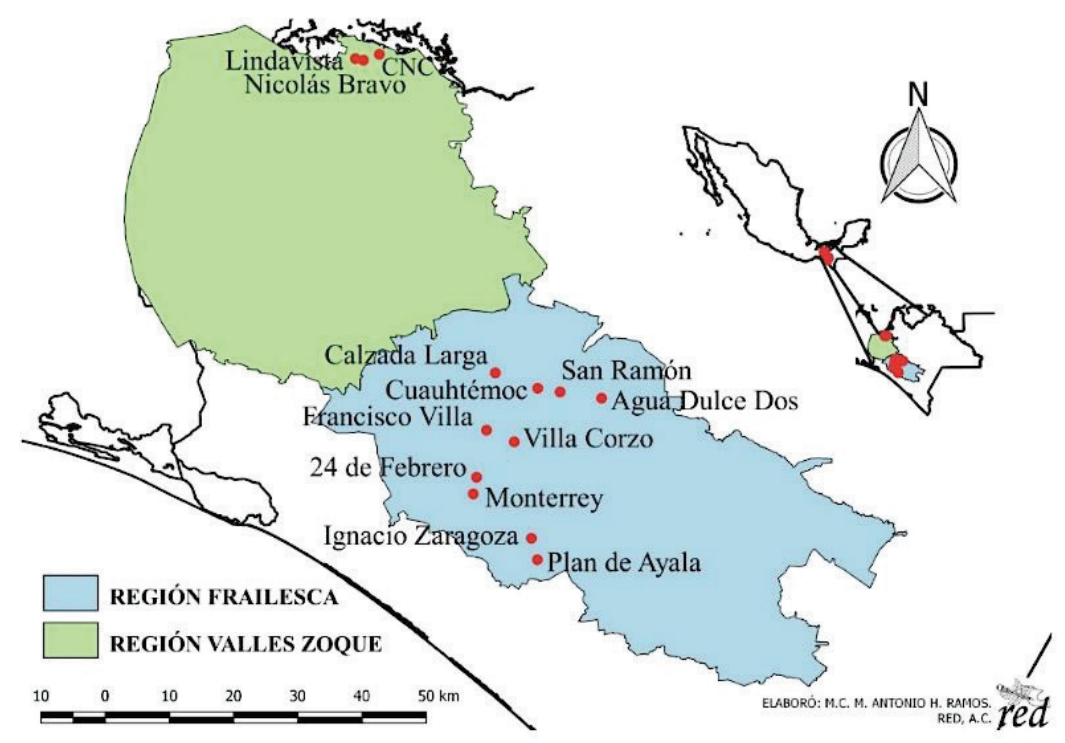

Figura 1. Localización de los sitios de muestreo en las regiones Frailesca y Valles Zoque, Chiapas, México.

Figure 1. Location of the sampling sites in Frailesca and Valles Zoque regions, Chiapas, Mexico. 
dedicados al maíz en dicha región (López-Báez et al., 2019), debido, entre otras causas, al uso de fertilizantes amoniacales, sobre todo sulfato y nitrato de amonio.

Se encontró un ajuste significativo a un polinomio de segundo grado entre la saturación por $\mathrm{Al}^{3+} \mathrm{y}$ el contenido de materia orgánica (Figura 2). La proporción de aluminio en el complejo de cambio del suelo aumentó proporcionalmente a la materia orgánica; sin embargo, a valores de materia orgánica por encima de $4.14 \%$ se produjo una disminución en el porcentaje de saturación de $\mathrm{Al}^{3+}$ en la solución del suelo, lo cual sugiere que pudiera ser la naturaleza de la materia orgánica y no su contenido lo que influyen en la acidez total de los ácidos húmicos y que en estos casos la materia orgánica forma complejos con el $\mathrm{Al}^{3+}$ proveniente de fuentes no determinadas y no tóxicas para las plantas (Ortiz-Escobar et al., 2004).

Esos resultados soportan los hallazgos de Villagrán-Manilla et al. (2019) quienes reportan que los ácidos húmicos y fúlvicos tienen una alta capacidad de complejar el $\mathrm{Al}^{3+}$, debido a la cantidad de grupos ácidos que al desprotonarse facilitan la formación de enlaces con el $\mathrm{Al}^{3+}$. De estos, el ácido fúlvico es uno de los compuestos que con mayor efectividad reduce la fitotoxicidad del aluminio, por su capacidad para formar complejos con monómeros y polímeros del elemento (Rivera et al., 2016).

De lo anterior se presupone que es posible lograr la reducción de los efectos tóxicos del $\mathrm{Al}^{3+}$ y mantener una nutrición equilibrada de las plantas cultivadas, mediante la aplicación de cal al suelo (Cristancho et al., 2011), ya que los carbonatos, óxidos, hidróxidos y silicatos de calcio o magnesio neutralizan los iones $\mathrm{H}^{+} \mathrm{y} \mathrm{Al}^{3+}$ en la solución del suelo (a través de los iones $\mathrm{OH}^{-}$) cuando la enmienda reacciona con el agua (Rivera et al., 2016).

Se corrobora entonces que el uso de las enmiendas o los materiales orgánicos, debido a su capacidad de mejorar la capacidad de intercambio catiónico, disminuyen la capacidad de retención de fósforo, favorecen la actividad microbiológica, mejoran las condiciones físicas y biológicas del suelo $\mathrm{y}$, en definitiva, aumentan el nivel de fertilidad del suelo (Rivera et al., 2016). Por otra parte, el calcio desplaza
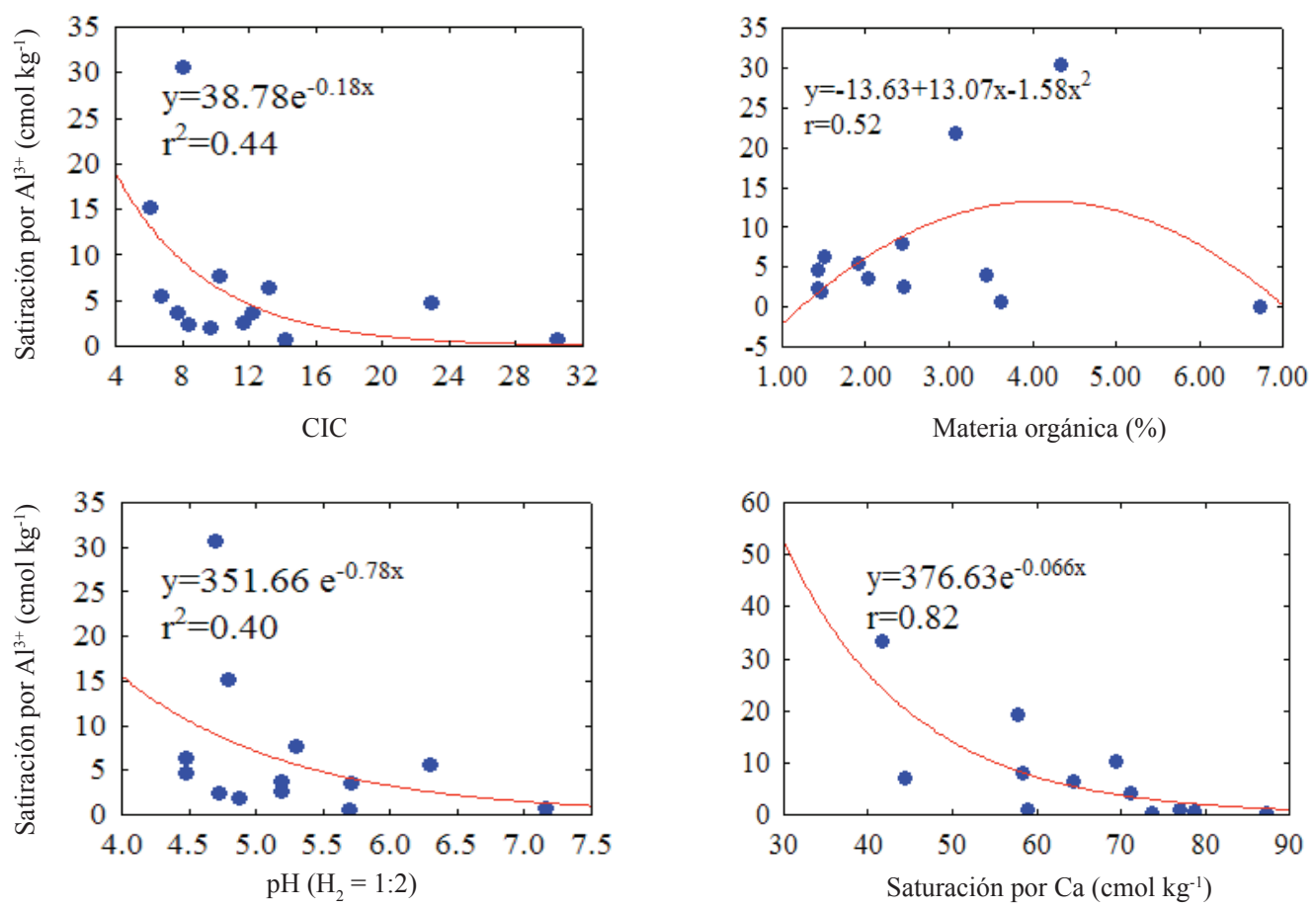

Figura 2. Efecto de la CIC (capacidad de intercambio catiónico), del contenido de materia orgánica, el pH y el Ca ${ }^{2+}$ sobre la saturación de $\mathrm{Al}^{3+}$ en suelos de las regiones Valles Zoques y La Frailesca, Chiapas. Figure 2. Effect of CIC (Cation-exchange capacity), organic matter content, $\mathrm{pH}$ and $\mathrm{Ca}^{2+}$ over $\mathrm{Al}^{3+}$ saturation in soils of the Valles Zoques and La Frailesca regions, Chiapas. 
al hidrógeno y el aluminio de los sitios de intercambio, con lo cual se logra aportar y liberar nutrientes retenidos en el suelo (Basak y Biswas, 2016; Rosas-Patiño et al., 2017).

Se encontró una relación negativa entre el hidrógeno intercambiable $\left(\mathrm{H}^{+}\right)$y la materia orgánica (Figura 3), demostrándose que conforme se incrementan los niveles de $\mathrm{MO}$, el $\mathrm{H}^{+}$intercambiable disminuye exponencialmente y que a valores por debajo de $2 \%$ de $\mathrm{MO}$, la saturación por $\mathrm{H}^{+}$puede alcanzar valores cercanos a $4.2 \mathrm{cmol} \mathrm{kg}^{-1}$ suelo $^{-1}$, equivalente a $20 \%$ de saturación por $\mathrm{H}^{+}$, lo cual provoca aumentos en la acidez intercambiable, ya que esta es el resultado de la presencia de hidrógeno $\left(\mathrm{H}^{+}\right)$y aluminio $\left(\mathrm{Al}^{+3}\right)$ que causan una disminución en el $\mathrm{pH}$. Sin embargo, hay que ser cautelosos a la hora de interceptar esas relaciones ya que se ha demostrado que cuando se establece la relación entre la acidez total y el contenido de materia orgánica no se ha encontrado relación alguna y el complejo materia orgánica-Al alcanza valores cercanos a cero, indicando que los altos contenidos de $\mathrm{Al}$ provienen de una fuente desconocida no tóxica para las plantas, que evita la aplicación de cal para corregir el aluminio (Ortiz et al., 2006).

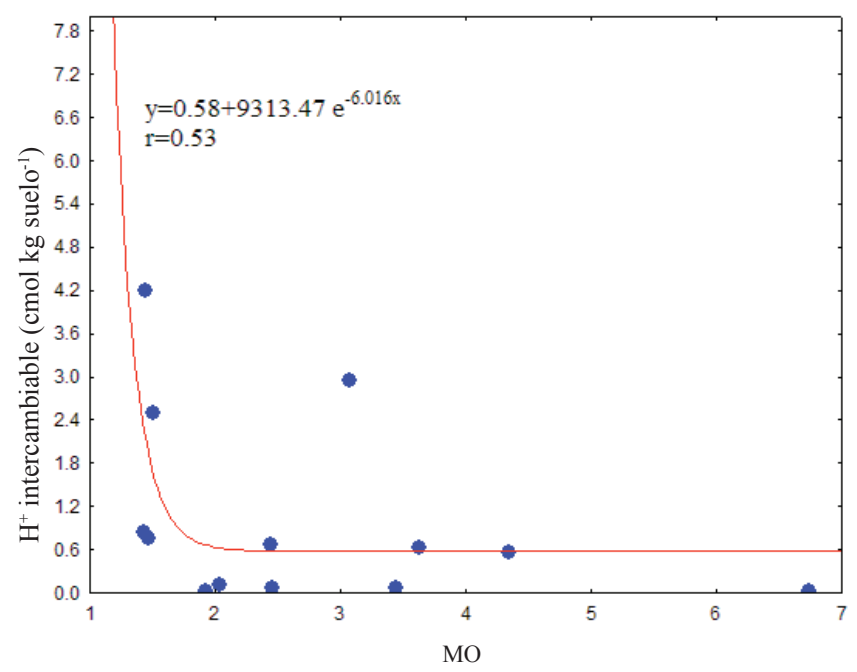

Figura 3. Efecto de la materia orgánica sobre la concentración de $\mathrm{H}^{+}$intercambiable en suelos de las regiones Valles Zoques y La Frailesca, Chiapas.

Figure 3. Effect of organic matter on the concentration of exchangeable $\mathrm{H}^{+}$in soils of the Valles Zoques and La Frailesca regions, Chiapas.

\section{CONCLUSIONES}

- La saturación de aluminio $\left(\mathrm{Al}^{3+}\right)$ en el complejo de intercambio de los suelos analizados no representa peligro de toxicidad para el cultivo de maíz y se correlacionó negativamente con la capacidad de intercambio catiónico, el $\mathrm{pH}$ y la saturación por $\mathrm{Ca}^{2+}$. Para el caso de la materia orgánica, valores por encima de $4 \%$ provocan disminuciones en la saturación por aluminio.

- La saturación por iones hidrógeno $\left(\mathrm{H}^{+}\right)$se correlacionó negativamente con la materia orgánica, indicando que valores por debajo de $2 \%$ de materia orgánica en los suelos, provoca incrementos en la acidez, probablemente debido a la disminución de la actividad microbiana que afecta la mineralización de la materia orgánica.

- En los suelos de la región Frailesca de Chiapas, se está produciendo un proceso de acidificación que pudiera conducir a incrementos tóxicos del aluminio para el cultivo de maíz, sobre todo a valores de $\mathrm{pH}$ inferiores a cinco. Tales incrementos en la acidificación de los suelos pudieran estar asociado al excesivo uso de fertilizantes amoniacales producto de una agricultura mucho más intensiva que en la región Valles Zoques.

\section{LITERATURA CITADA}

Basak, B. B. and D. R. Biswas. 2016. Potentiality of Indian rock phosphate as liming material in acid soil. Geoderma 263: 104109. doi: http://dx.doi.org/10.1016/j.geoderma.2015.09.016.

Cristancho, J. A., M. M. Hanafi, S. R. Syed Omar, and M. Y. Rafii. 2011. Alleviation of soil acidity improves the performance of oil palm progenies planted on an acid Ultisol. Acta Agric. Scand. 61: 487-498. doi: https://doi.org/10.1080/09064710.2 010.506448 .

INEGI (Instituto Nacional de Estadística y Geografía). 2017. Anuario estadístico y geográfico de Chiapas 2017. Gobierno del Estado de Chiapas. http://internet.contenidos.inegi.org.mx/ contenidos/Productos/prod_serv/contenidos/espanol/bvinegi/ productos/nueva_estruc/aegef_2017/702825097929.pdf (Consulta: febrero 20, 2019).

Lal, R. 2015. Restoring soil quality to mitigate soil degradation. Sustainability 7: 5875-5895. doi: https://doi.org/10.3390/ su7055875.

López Báez, W., R. Reynoso-Santos, J. López-Martínez, B. VillarSánchez, R. Camas-Gómez y J. García-Santiago. 2019. Caracterización físico-química de suelos cultivados con maíz en Villaflores, Chiapas. Rev. Mex. Cienc. Agríc. 10: 897-910. doi: https://doi.org/10.29312/remexca.v10i4.1764. 
Lupwayi, N. Z., M. B. Benke, X. Hao, J. T. O’Donovan, and G. W. Clayton. 2014. Relating crop productivity to soil microbial properties in acid soil treated with cattle manure. Agron. J. 106: 612-621. doi: https://doi.org/10.2134/agronj2013.0427.

Montejo-Martínez, D., F. Casanova-Lugo, M. García-Gómez, I. Oros-Ortega, V. Díaz-Echeverría y E. R. Morales-Maldonado. 2018. Respuesta foliar y radical del maíz a la fertilización biológica-química en un suelo Luvisol. Agron. Mesoam. 29: 325-341. doi: 10.15517/ma.v29i2.29511.

Muhammad, N., G. Zvobgo, and G. Zhan. 2019. A review: The beneficial effects and possible mechanisms of aluminum on plant growth in acidic soil. J. Integr. Agric. 18: 1518-1528. doi: https://doi.org/10.1016/S2095-3119(18)61991-4.

Ortiz-Escobar, M. E., R. D. Zapata H., S. Sadeghian-Khalajabadi y H. F. Franco A. 2004. Aluminio intercambiable en suelos con propiedades ándicas y su relación con la toxicidad. Cenicafé 55: 101-110.

Ortiz-Escobar, M. E., R. D. Zapata y S. Sadeghian-Khalajabadi. 2006. Propiedades de la materia orgánica y capacidad complejante sobre el aluminio en algunos suelos ándicos en Colombia. Cenicafé 57: 51-57.

Rivera, Y., L. Moreno, M. Herrera y H. M. Romero. 2016. La toxicidad por aluminio $\left(\mathrm{Al}^{3+}\right)$ como limitante del crecimiento y la productividad agrícola: el caso de la palma de aceite. Palmas 37: $11-23$.
Rosas-Patiño, G., Y. J. Puentes-Páramo y J. C. Menjivar-Flores. 2017. Relación entre el $\mathrm{pH}$ y la disponibilidad de nutrientes para cacao en un entisol de la Amazonia colombiana. Corpoica Cienc. Tecnol. Agropec. 18: 529-541. doi: https://doi. org/10.21930/rcta.vol18_num3_art:742.

SEMARNAT (Secretaría de Medio Ambiente y Recursos Naturales). 2002. Norma Oficial Mexicana NOM-021SEMARNAT-2000, que establece las especificaciones de fertilidad, salinidad y clasificación de suelos, estudio, muestreo y análisis. SEMARNT. México, D. F.

StatSoft, Inc. 2015. STATISTICA (data analysis software system), version 12.5 www.statsoft.com.

Villagrán-Manilla, A., E. D. Ibarra-Coria, M. E. Páez-Hernández y S. Nieto-Velázquez. 2019. Evaluación y análisis de cinéticas de agregación de los ácidos húmicos en presencia de $\mathrm{Al} 3+$ por espectroscopia UV-VIS. Tópicos Invest. Cienc. Tierra Mat. 6: 72-79. doi: https://doi.org/10.29057/aactm.v6i6.5000.

Wan, W. J., J. Tan, Y. Wang, Y. Qin, H. He, H. Wu, W. Zuo, and D. He. 2019. Responses of the rhizosphere bacterial community in acidic crop soil to $\mathrm{pH}$ : Changes in diversity, composition, interaction, and function. Sci. Total Environ. 697: 134418. doi: https://doi.org/10.1016/j.scitotenv.2019.134418.

Zhao, W., J. Li, J. Jianga, H. Lua, Z. Honga, W. Qiana, R. Xua, K. Denga, and P. Guana. 2020. The mechanisms underlying the reduction in aluminum toxicity and improvements in the yield of sweet potato (Ipomoea batatas L.) after organic and inorganic amendment of an acidic ultisol. Agric. Ecosyst. Environ. 288: 106716. doi: https://doi.org/10.1016/j.agee.2019.106716. 